\title{
Valoración Económica Para La Conservación Del Bosque De La Cuenca De Tecocomulco, Hidalgo
}

\section{Economic Valuation For Conservation Of The Forest Of The Watershed Tecocomulco, Hidalgo}

\author{
Ramos Álvarez, María de Jesús; Larqué Saavedra, Bertha Sofía; \\ Hernández-Ortíz, Juan; Monroy-Hernández, Rubén; Hernández- \\ Álvarez, Zenón; Editor Académico Dr. Prof Carlos A. Zúniga- \\ González
}

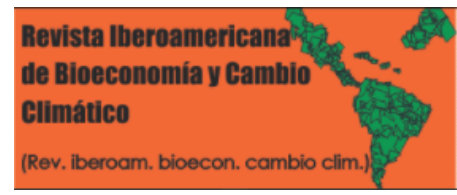

María de Jesús Ramos Álvarez

mariramos_al@hotmail.com

Universidad Autónoma Chapingo, México

Bertha Sofía Larqué Saavedra

larque.bertha@inifap.gob.mx

Instituto Nacional de Investigaciones Forestales

Agrícolas y Pecuarias, México

(iD) Juan Hernández-Ortíz

jhdzo@yahoo.com.mx

Universidad Autónoma Chapingo, México

(iD) Rubén Monroy-Hernández

ruben.monroy@unach.mx

Universidad Autónoma de Chapingo, México

Zenón Hernández-Álvarez zenon.hernandez@conafor.gob.mx

Comisión Nacional Forestal. Cintalapa de Figueroa

Chiapas, México

Editor Académico Dr. Prof Carlos A. Zúniga-

González

Universidad Nacional Autónoma de Nicaragua, León,

Nicaragua

Revista Iberoamericana de Bioeconomía y Cambio Climático

Universidad Nacional Autónoma de Nicaragua, León, Nicaragua ISSN-e: 2410-7980

Periodicidad: Semestral

vol. 7, núm. 13, 2021

czuniga@ev.unanleon.edu.ni

Recepción: 26 Enero 2021

Aprobación: 11 Mayo 2021

URL: http://portal.amelica.org/ameli,

jatsRepo/394/3941760010/index.html

DOI: https://doi.org/10.5377/ribcc.v7i13.11421

Autor de correspondencia: jhdzo@yahoo.com.mx
Resumen: En el presente estudio se realizó una valoración económica aplicando el método de valoración contingente para la conservación del bosque de la cuenca Laguna de Tecocomulco. Se eligió como caso de estudio los municipios de Almoloya, Apan y Tepeapulco. Se aplicaron 266 cuestionarios determinado por muestreo simple aleatorio y muestreo proporcional. Los resultados muestran que la DAP promedio fue de $\$ 13.33$ mensual por persona obtenido mediante el modelo logit binomial, el valor económico para la conservación del bosque se estimó en \$539,111.41 mensual. El 65\% de los encuestados están dispuestos a pagar para conservar el bosque. El $85 \%$ conocen los beneficios que provee el bosque y el $88 \%$ están conscientes del deterioro ambiental que existe en la cuenca. Las variables que resultaron estadísticamente significativas con un nivel de confiabilidad del $95 \%$, en la determinación de la DAP son: edad, educación, género y conocimiento sobre los beneficios del bosque.

Palabras clave: Método de valoración contingente, Disposición a pagar, Regresión logística.

\begin{abstract}
In the present study, an economic valuation was realized by using the contingent valuation method. This was done to aid the conservation of the forest of the watershed lagoon of Tecocomulco. The municipalities of Almoloya, Apan and Tepeapulco were chosen as a case study. 266 questionnaires were applied, determined by simple random sampling and proportional sampling. The results show than the WTP average was $\$ 13.33$ monthly per person. This result was obtained by the binomial logit model. The economic value for the conservation of the forest was estimated at $\$ 539111.41$ monthly. $65 \%$ of the respondent are willing to pay to preserve the forest, $85 \%$ know of the benefits by forests and $88 \%$ are aware of the environmental degradation that exists in the watershed. The variables that were statistically significant at a confidence level of $95 \%$ in the determination of the WTP are: age, education, gender and knowledge about the benefits of forest.
\end{abstract}

Keywords: Contingent valuation method, Willingness to pay, Logistic regression. 


\section{INTRODUCCIÓN}

Las actividades que impulsan el desarrollo de la sociedad ejercen una fuerte presión sobre los ecosistemas forestales y, afectando a las especies que los integran, su estructura y la persistencia y calidad de los servicios ambientales que brindan. En varias zonas del país se está dando el cambio de zonas agrícolas por zonas urbanas, provocando la disminución de los servicios ambientales de los terrenos agrícolas. Lo mismo sucede con la práctica de una agricultura intensiva. El cambio de uso del suelo, la sobreexplotación y uso ilegal de los recursos naturales, los incendios forestales son los principales factores de amenaza de los ecosistemas.

De acuerdo con un informe de SEMARNAT (2016), entre 1993 y 2002, México perdió cerca de 2.5 millones de hectáreas de bosques, 837 mil de matorrales xerófilos, 836 mil de selvas y 95 mil hectáreas de humedales. Las altas tasas de deforestación (bosques y selvas) han ocasionado pérdidas de los servicios ambientales a nivel de cuenca. Desde el punto de vista económico, una cuenca hidrográfica provee a la sociedad diversos bienes y servicios comercializables y no comercializables. Entre los comercializables se encuentra principalmente el agua que sirve como base de sistemas de producción local de las personas que viven dentro del límite de la cuenca y los no comercializables (servicios ambientales). Landell-Mills y Porras (2002) identificaron los siguientes servicios ambientales: función de regulación de flujos de agua, tanto en temporada de sequía como el control de inundaciones; mantenimiento de la calidad del agua (control de sedimentos en las partes medias y bajas de la cuenca y control de salinidad); control de erosión; salinización del suelo (relación agua/salinidad); mantenimiento de hábitats acuáticos. En los últimos años, en la parte centro del país existe una mayor demanda de agua ocasionado por el crecimiento poblacional, por ello la relación entre bosque y cuenca hidrológica, cobra mayor importancia.

En la parte alta y media de la cuenca laguna de Tecocomulco se presenta problemas de deforestación, provocando pérdida de cobertura vegetal y arrastre de sedimentos, lo cual implica desertificación de los terrenos. Por otro lado, en la parte baja de la cuenca se identifica problemas de azolvamiento de los causes de la laguna provocando inundaciones de los cultivos agrícolas y con ello perdidas en la producción, según Martínez (2011) el aporte de sedimentos a la laguna es de 248,000 toneladas anual.

En base a la información proporcionada por CONAGUA (2009), la laguna de Tecocomulco es considera un acuífero subexplotado, por lo que actualmente, el problema a combatir es la deforestación de la parte alta de la cuenca, lo que implica que el suelo se quede descubierto, siendo vulnerable al viento y a la lluvia. Es importante hacer trabajos para revertir estos problemas, sino en un futuro el arrastre de sedimentos impactara en la pérdida de la capacidad de almacenamiento, representando un problema social, pues al reducirse la capacidad de almacenamiento provocara inundaciones masivas sobre todo en la parte de la cuenca baja.

Con el propósito de contribuir en la búsqueda de posibles soluciones a la problemática de la laguna, la presente investigación se planteó con el objetivo de realizar una valoración económica para la conservación del bosque por parte de los usuarios de la cuenca laguna de Tecocomulco, a través del método de valoración contingente. Los objetivos específicos para esta investigación fueron: determinar la disposición a pagar por los beneficiarios del bosque de la cuenca hidrológica laguna de Tecocomulco; identificar que variables influyen en la disposición a pagar para la conservación del bosque y caracterizar a la población beneficiaria de los servicios que provee el bosque. Se pretende que este estudio sea un referente para los funcionarios del gobierno encargado de elaborar políticas públicas a cuantificar en términos económicos la importancia relativa de servicios ambientales generados por los bosques dentro de una cuenca hidrográfica, diseños de programas en

Notas DE AUTOR

jhdzo@yahoo.com.mx 
el sector ambiental, así también para analizar las políticas y programas actuales, que se están implementado, dentro del ámbito local.

Existen estudios que se han enfocado a la valoración económica de los servicios ambientales. Empleando el método de valoración contingente, Saldívar et. al, (2013), realizaron un estudio para estimar la disposición a pagar por partes de los usuarios domésticos del servicio ambiental hidrológico en el Parque Nacional Cumbres de Monterrey, la estimación de la DAP de la población de la Zona Metropolitana de Monterrey reveló que la media de pago es de $\$ 6.80$ mensuales, los autores indican que, aplicar un pago generalizado de cinco pesos por mes, por cada toma domiciliaria sería aceptado por la población; donde el recibo de agua potable sería el medio de pago propuesto por los encuestados.

Del Ángel et. al, (2009), estudiaron la disposición a pagar del servicio ambiental hidrológico para uso doméstico de San Andrés Tuxtla, Veracruz, México, a través del método de valoración contingente. Los resultados obtenidos fueron: el $88 \%$ de los encuestados están dispuesto a pagar, la DAP promedio fue de $\$ 6.02$ mensual, teniendo como medio de pago el recibo de agua.

\section{MATERIALES Y MÉTODOS}

Se trabajó en los municipios de Apan, Almoloya y Tepeapulco del estado de Hidalgo, considerando que se localizan en la parte baja de la cuenca, pues son los que sufren las consecuencias de la deforestación del bosque, tales como inundaciones en los cultivos agrícolas. Se empleó el método de valoración contingente, los materiales y herramientas que se utilizaron fueron encuestas y programas computacionales tales como $\mathrm{N}$ Logit versión 4.0, y Microsoft Office 2010.

\section{Determinación de la muestra}

El tamaño de la muestra ec1 se calculó a partir de la población económicamente activa registrada en el censo de población 2018, a través de un muestreo aleatorio simple (MAS). La muestra se conformó de 266 cuestionarios, para ello se aplicó la fórmula siguiente:

$$
n=\frac{\mathrm{N}}{1+\frac{e^{2}(\mathrm{~N}-1)}{\mathrm{Z}^{2} p q}}
$$

$\mathrm{n}=$ Tamaño de la muestra

$\mathrm{N}=0,418$ (Población económicamente activa de los tres municipios)

$\mathrm{e}=$ Error aceptado $(0.06)$

$p q=0.25$

$\mathrm{Z}_{\alpha / 2}=$ Confianza de $95 \%$

Para determinar el tamaño por municipio se utilizó la expresión ec2 siguiente:

$$
n_{h}=\frac{(n) *(h)}{\mathrm{N}}
$$

Donde:

$n_{h}$, Muestra por municipio

$h$, Población económicamente activa por municipio

De esta manera la muestra quedó definida como sigue: 


$$
\begin{aligned}
& n_{\mathrm{A} \text { lmoloya }}=[266 * 4120] / 40418=27 \\
& n_{\mathrm{A} \text { pan }}=[266 * 16046] / 40418=106 \\
& n_{\text {Tepeapulco }}=[266 * 20252] / 40418=133
\end{aligned}
$$

\section{Diseño y aplicación del cuestionario}

Se diseñó un cuestionario con tres secciones. La primera incluyó la percepción y conocimiento sobre los recursos naturales que el bosque provee. La segunda fue de valoración de los servicios ambientales del bosque, considerando como pregunta básica ¿Está dispuesto a pagar por conservar el bosque? ¿Cuánto está dispuesto a pagar mensual? Y la tercera sección se preguntó información sobre las características socioeconómicas del encuestado. Para la aplicación de cuestionario, se eligieron personas mayores de 18 años asumiendo que tienen decisiones propias y económicamente independientes, capaces de emitir un juicio sobre la protección del bosque.

\section{Método de valoración contingente (MVC)}

El método de valoración contingente (MVC) busca estimar la disposición a pagar o aceptar simulando un mercado mediante aplicación de encuesta a los consumidores potenciales de los servicios ambientales. Se les pregunta por la máxima cantidad de dinero que pagarían por el bien si tuvieran que comprarlo, como hacen con los demás bienes o la cantidad mínima dispuestos a aceptar con tal de renunciar a un beneficio individual en deterioro del ambiente, de esta forma se deduce el valor que para el consumidor tiene el bien en cuestión (Riera, 1994).

Hanemann (1984) desarrolló formulaciones teóricas sobre el MVC con formato binario. Considerando la maximización de la utilidad del consumidor cuando se incorpora la demanda por servicios ambientales, desde el punto de vista la teoría microeconómica, la función indirecta de utilidad está dada por:

$$
u_{j}=v_{j}\left(p, y ; z_{j}\right)
$$

Donde:

$j=0$, Es la situación inicial y $j=1$, Es la situación modificada

$p$,Es el vector de precios,

$z_{j}$, Es el vector de calidad bienes ambientales y/o características socioeconómicas del individuo.

Debido al supuesto principal de valoración contingente, de que las funciones de utilidad tienen componentes que el investigador desconoce; lo cual sirve para generar una estructura estocástica para la función de utilidad (ec. 3). De esta manera se considera la función indirecta de utilidad es una variable aleatoria con alguna distribución de probabilidad para los parámetros con medias que dependen de las características observables del individuo, por lo que la ecuación 3, (ec4) se puede reescribir como:

$$
u_{j}=v_{j}\left(p, y ; z_{j}\right)+\varepsilon_{j}
$$

$j=0,1$

Donde $\varepsilon_{0}$ y $\varepsilon_{1}$ son variables aleatorias independientes e idénticamente distribuida (i.i.d) con media cero. La probabilidad de una respuesta positiva por parte del individuo, está dada por la siguiente expresión (ec 5): 


$$
\begin{gathered}
\operatorname{Pr} \square \mathrm{si} \square=\operatorname{Pr}\left[u_{1} \square \mathrm{p}, \mathrm{y}-\mathrm{At} ; \mathrm{z} \square+\varepsilon_{1}>\mathrm{u}_{0} \square \mathrm{p}, \mathrm{y} ; \mathrm{z}_{0} \square+\varepsilon_{0}\right] \\
\operatorname{Pr} \square \mathrm{si} \square=\operatorname{Pr}\left[\mathrm{u}_{1} \square \mathrm{p}, \mathrm{y}-\mathrm{At} ; \mathrm{z}_{1} \square>\mathrm{u}_{0} \square \mathrm{p}, \mathrm{y} ; \mathrm{z}_{0} \square>\varepsilon_{0}-\varepsilon_{1}\right] \\
\operatorname{Pr} \square \mathrm{si} \square=\operatorname{Pr}\left[\Delta u>\varepsilon_{0}-\varepsilon_{1}\right] \\
\operatorname{Pr} \square \mathrm{si} \square=\operatorname{Pr}[\Delta u>\eta] \\
\operatorname{Pr}(\mathrm{si})=\operatorname{Fn}(\Delta u)
\end{gathered}
$$

Donde $\mathrm{F}_{n}$ es la función de distribución acumulada de $\eta \mathrm{y} \eta=\varepsilon_{0}-\varepsilon_{1}$. Al elegir una distribución para $\eta$, y especificando apropiadamente $v\left(\right.$.) , los parámetros de la diferencia indicada por $\Delta_{v}$ pueden ser estimados con la cantidad de disponibilidad a pagar de los individuos generados a través de la respuesta a la pregunta binaria y la información sobre las características socioeconómicas de los encuestados.

La forma pragmática para la función indirecta de utilidad propuestas por Hanemann (1984) y Bishop y Herberlein (1979) son (Cuadro 1):

\begin{tabular}{lcc}
\hline Nombre & Función $v$ & Forma funcional \\
\hline Función indirecta de utilidad lineal & $v_{i}=\alpha_{i}+\beta y+\varepsilon_{i}$ & $\Delta v=\alpha-\beta A_{t}$ \\
Función indirecta de utilidad de Bishop & $v_{0}=y+\delta$ & $\Delta v=\alpha-\beta \ln A_{t}$ \\
y Herbelein & $v_{1}=y+\delta+\exp \frac{\alpha+\varepsilon}{\beta}$ & \\
& & \\
\hline
\end{tabular}

\section{CUADRO 1}

Funciones de utilidad.

Vásquez et al, 2007.

La función indirecta de utilidad lineal se representa con la siguiente expresión ec 6:

$$
v_{i}=\alpha_{i}+\beta y+\varepsilon_{i} \beta>0 y i=0,1
$$

Donde $\alpha$ y $\beta$ son funciones del vector de calidad bienes ambientales y/o características socioeconómicas del individuo $(z)$. Por lo que se puede expresar para la situación inicial como ec 7:

$$
v_{0}=\alpha_{0}+\beta y+\varepsilon_{0}
$$

Y la situación final se puede escribir de la siguiente manera ec 8:

$$
\begin{gathered}
u_{1}=a_{1}+\beta\left(y-\mathrm{A}_{\mathrm{t}}\right)+\varepsilon_{1} \\
u_{1}=a_{1}+\beta(y-\mathrm{DAP})+\varepsilon_{1}
\end{gathered}
$$

El cambio de la función de utilidad queda descrito como ec 9:

$$
\begin{gathered}
\Delta u=a_{1}+\beta(y-\beta \mathrm{DAP})-\left(\alpha_{0}+\beta y\right) \\
\Delta u=\left(a_{1}-\alpha_{0}\right)-\beta \mathrm{DAP} \\
\Delta v=a-\beta \mathrm{DAP}
\end{gathered}
$$


La función de distribución de probabilidad es:

$\mathrm{P}=F n\left(\Delta_{v}\right)=\left(1+e^{-(\alpha-\beta \mathrm{DAP})}\right)-1$

En la función indirecta de utilidad de Bishop y Herbelein, el cambio en la función de utilidad está dada por ec10:

$$
\Delta_{v}=a-\beta \operatorname{lnC}+\eta
$$

Donde C que representa la DAP es definitiva por: $\ln C=\frac{\alpha+\eta}{\beta}$ entonces $\mathrm{C}=\varepsilon_{\varepsilon} \frac{a}{\beta} \frac{\eta}{\beta}$

A partir de estas funciones de utilidad es posible calcular la DAP, cuando se utiliza la función indirecta de utilidad lineal, la media y la mediada se obtiene de la misma forma, donde ec11:

$$
\mathrm{DAP}_{\text {media }}=\frac{\alpha}{\beta}
$$

En el caso de función indirecta de utilidad de Bishop y Herberlein el cálculo de la mediana es a través de la formula siguiente ec 12 :

$$
\mathrm{DAP}_{\text {media }}=e\left(\frac{a}{\beta}\right)
$$

Y la media con la expresión ec 13:

$$
\mathrm{DAP}_{\text {medli }}=e\left(\frac{a}{\beta}\right) \mathrm{E}\left(e\left(\frac{\eta}{\beta}\right)\right)
$$

Donde $E\left(e^{\frac{\eta}{\beta}}\right)=\frac{\pi}{\beta^{*} \operatorname{sen} 0\left(\frac{\pi}{\beta}\right)}$

Cuando el modelo estima valores negativos de la DAP se requiere aplicar un modelo restringido, una forma de corregir los DAP negativa de acuerdo Haab y McConnell (2002) es truncar la E [DAP ] entre cero y el monto máximo a pagar declarado por el encuestado. Una manera directa de derivar un modelo que limite la DAP correctamente, se presenta en la expresión siguiente ec 14:

$$
\mathrm{DAP}_{j}=\mathrm{G}\left(\mathrm{Z}_{j Y}+\varepsilon_{j}\right) y_{j}
$$

Donde $0 \leq G\left(Z_{i \gamma}+\varepsilon_{j}\right) \leq 1$ y $\mathrm{G}^{\prime}\left(\mathrm{Z}_{i \gamma}+\varepsilon_{j}\right) \geq 0$. La función $\mathrm{G}\left(\mathrm{Z}_{j \gamma}+\varepsilon_{j}\right)$ es la DAP. La versión específica del modelo es ec 15:

$$
\operatorname{DAP}_{j}=\frac{\text { Montomax }}{1+e^{\left(-Z_{\left.j \gamma^{-\varepsilon}\right)}\right.}}
$$

Si el $\varepsilon$ se distribuye logísticamente con $\left(0, \sigma^{2}\right)$ entonces la probabilidad es definida por ec 16 : 


$$
\operatorname{Pr}(s i)=1-\mathrm{F}_{\mathrm{DAP}(t)}=\frac{1}{1+e^{-\left[\mathrm{Z}_{j \gamma}-\ln \left(\frac{\mathrm{Montomax}}{\text { monto }}\right)\right] / \sigma}}
$$

Y la media se calcula como ec 17 :

$$
\mathrm{E}(\mathrm{DAP})=\frac{\text { Montomax }}{1+e^{-Z_{j}\left(\frac{a}{\beta}\right)}}
$$

\section{Modelo econométrico}

De acuerdo Riera (1994) la DAP se ajusta una distribución de probabilidad normal o logística. La distribución logit o logística, es una de las más utilizadas en estudios de valoración contingente. En el modelo logit de máxima verosimilitud, asumiríamos que ec 18:

$$
\mathrm{Y}_{i}=\alpha+\beta_{k} \mathrm{X}_{\kappa i}+\varepsilon_{i}
$$

$\mathrm{Y}_{i}$ donde esta variable Es inobservable, pero se manifiesta a través del indicador de la variable discreta,

$\mathrm{Y}_{i}=\left\{\begin{array}{l}\text { 1sicurreelevento } \\ \text { 0encasocontrario }\end{array}\right.$

$\mathrm{X}_{k i}$ son las variables explicativa

$\varepsilon_{i}$ es la variable aleatoria que se distribuya normal $\mathrm{N}\left(0, \sigma^{2}\right)$

Su característica principal de éste modelo, es que las observaciones se dividen en dos grupos. En el primer grupo se encuentra las observaciones que donde sí ocurrió el evento esperado $\left(\mathrm{Y}_{i}=1\right)$, es la respuesta afirmativa de disposición al pago por conservar el bosque. El segundo grupo es el evento que no ocurrió $\left(\mathrm{Y}_{i}=0\right)$, es decir, un DAP negativa. El modelo logit se basa en la función logística ec 19:

$$
\mathrm{F}_{z}=\frac{1}{1+e^{-z}}=\frac{e^{z}}{1+e^{z}}
$$

Las probabilidades de que la variable independiente tome el valor de 1 y 0 son ec 20 y ec 21 :

$$
\begin{aligned}
& P(Y=1)=E\left(Y_{i} \mid X=x\right)=\frac{e^{z i}}{1+e^{z i}} \\
& P(Y=0)=1-\frac{e^{z i}}{1+e^{z i}}=\frac{1}{1+e^{z i}}
\end{aligned}
$$

Donde $Z_{i}=\beta_{1}+\beta_{2} x_{2 i}+\cdots+\beta_{k} x_{k i}$

El método utilizado para estimar los parámetros del modelo, es el de Máxima verosimilitud. La interpretación de los parámetros estimados en el modelo logit, así como el signo correspondiente a cada parámetro indica la relación que guarda las variables explicativas con la variable dependiente, siendo la variable dependiente la DAP, es decir, indica si la probabilidad aumenta o disminuye cuando aumenta la 
variable explicativa correspondiente o viceversa. Es importante tener en cuenta que el modelo logit parte del supuesto de una relación no lineal entre las variables explicativas y la probabilidad de ocurrencia del evento, cuando aumenta en una unidad la variable explicativa los incrementos en la probabilidad normalmente no son iguales.

Para estimar la cantidad monetaria destinada a la conservación de los servicios ambientales del bosque, se analizó la variable DAP como dependiente en función de variables socioeconómicas y de percepción ambiental: monto a pagar, ingreso, edad, educación, percepción de problemas ambientales y conocimiento sobre los beneficios que genera el bosque. De acuerdo a Valdivia et. al, (2012), los softwares utilizados para modelos de elección binaria son: N-logit, SAS, Stata. En este estudio se empleó N-logit versión 4.0, el modelo econométrico se expresó como:

Modelo logit tradicional

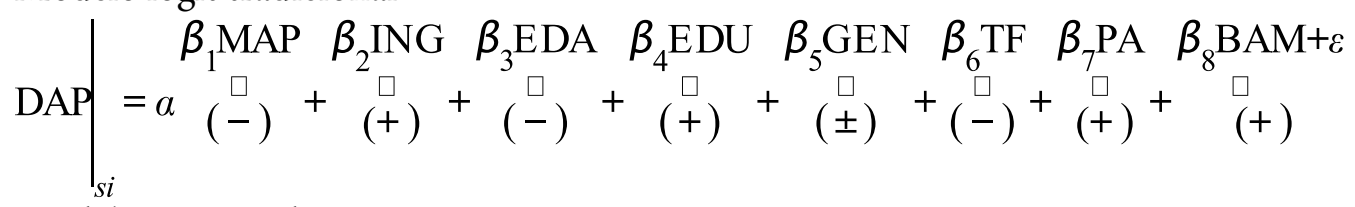

Modelo restringido

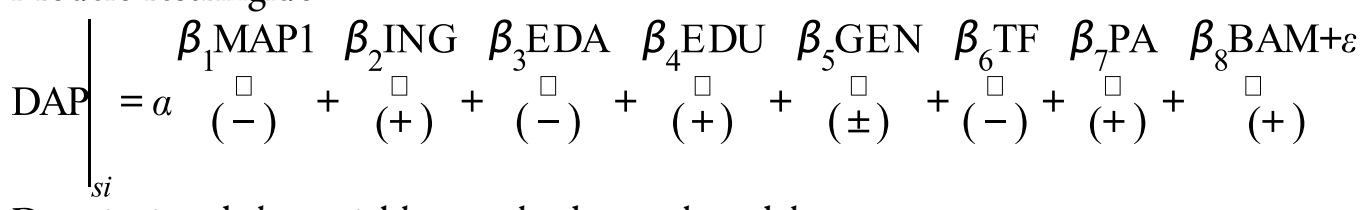

Descripcion de las variables empleadas en el modelo propuesto.

DAP. Probabilidad de que el encuestado(a) responda afirmativamente; variable dependiente dicotómica; se codificó como: $\mathrm{SI}=1$ si está dispuesto a pagar, $\mathrm{NO}=0$, no está dispuesto a pagar.

BAM. Indica si el encuestado(a) tiene o no conocimiento sobre los beneficios que genera el bosque, variable dicotómica; se codificó como: $\mathrm{SI}=1$, tiene conocimiento, $\mathrm{NO}=0$, no tiene conocimiento.

PA. Indica la percepción del deterioro ambiental; variable independiente dicotómica; se codificó como: $\mathrm{SI}=1$, existe deterioro ambiental, $\mathrm{NO}=0$, no existe deterioro ambiental.

GEN. Representa el género del encuestado(a); variable dicotómica, se codificó como: Hombre=1, Mujer $=0$.

TF. Representa el número de personas que viven en el hogar, variable ordenada categóricamente; se codificó como: 1 a 3 personas=1, 4 a 6 personas=2, 7 a 11 personas $=3$.

EDU. Representa el nivel educativo del encuestado(a); variable ordenada categóricamente, se codificó como: No fue a la escuela $=0$, Primaria $=1$, Secundaria $=2$, Preparatoria $=3$, Licenciatura $=4$.

EDA. Variable que indica la edad de la encuestada (a), ordenada categóricamente, se codificó como: 18 a 25 años $=1 ; 26$ a 35 años $=2 ; 36$ a 35 años $=3 ; 46$ a 60 años $=4$; Mayor de 60 años $=5$.

ING. Representa el ingreso mensual del encuestado(a); variable ordena categóricamente, se codificó como: Menos de $\$ 4,000.00=1, \$ 4001.00$ a $\$ 6,000.00=2 ; \$ 6,001.00$ a $\$ 8,000.00=3 ; \$ 8,001.00$ a $\$ 10,000.00=4 ; \$ 10,001.00$ a $\$ 12,000.00=5 ; \$ 12,001.00$ a $\$ 14,000.00=6 ; \$ 14,000.00$ a $\$ 16,000.00=7$; Más de $\$ 16,000.00=8$.

MAP. Cantidad mensual en pesos que el encuestado(a) está dispuesto a pagar para conservar el bosque, variable continua.

MAP1. Variable transformada de la MAP. Representa el logaritmo natural de la MAP acotado por la cantidad máxima.

$\varepsilon$ Es el termino de error; $\beta$ los parámetros a estimar y la constante de la función. 


\section{RESULTADOS Y DISCUSIÓN}

\section{Análisis descriptivo}

Entre las variables socioeconómicas importantes se puede destacar la edad, la educación y el ingreso de la población objetiva.

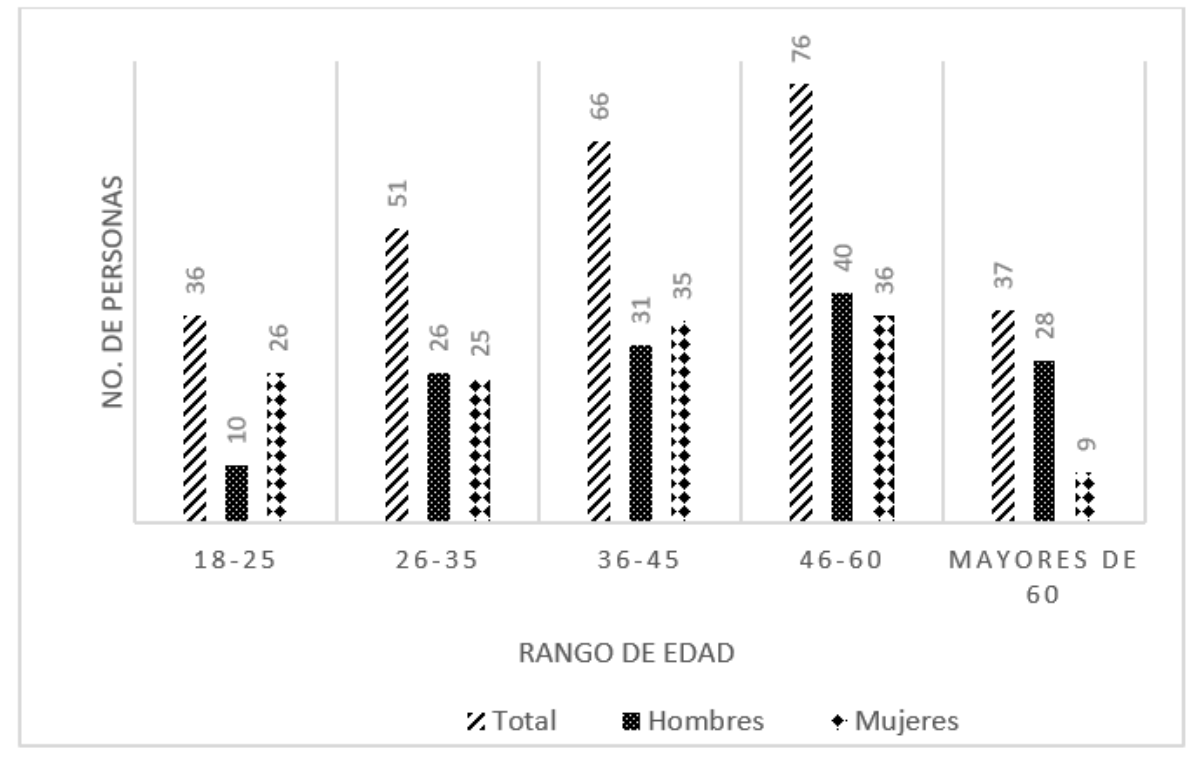

FIGURA 1 .

Edad de la población por rango.

Elaboración con datos de las encuestas.

La edad del $53.4 \%$ (142 personas) de población encuestada pertenecían al grupo de edad entre 36 y 60 años, personas que formaban parte de la población económicamente activas y con poder de decisión propias; el 13.5\% tenían entre los 18 y 25 años de edad y el 13.9\% (37 encuestados) eran mayores de 60 años (figura 1).

En cuanto al nivel educativo de los encuestados, se obtuvo que la mayoría de la población, al menos fue a la primaria, solo se reportaron 6 casos donde las personas no fueron a la escuela representando el $2 \%$ de la población muestral; el 15\% cursó el nivel de primaria, 32\% secundaria, 36\% preparatoria y el 13.5\% estudió licenciatura (Cuadro 2).

\section{CUADRO 2 .}

Escolaridad de la población.

\begin{tabular}{lllll}
\hline Nivel & $\begin{array}{l}\text { No. De } \\
\text { casos }\end{array}$ & Porcentaje & Hombres & Mujeres \\
\hline Sin estudios & 6 & 2.26 & 3 & 3 \\
Primaria & 41 & 15.41 & 29 & 12 \\
Secundaria & 86 & 32.33 & 39 & 47 \\
Preparatoria & 97 & 36.46 & 43 & 53 \\
Licenciatura & 36 & 13.53 & 20 & 16 \\
TOTAL & 266 & 100.00 & 135 & 131 \\
\hline
\end{tabular}

Elaboración con datos de las encuestas 


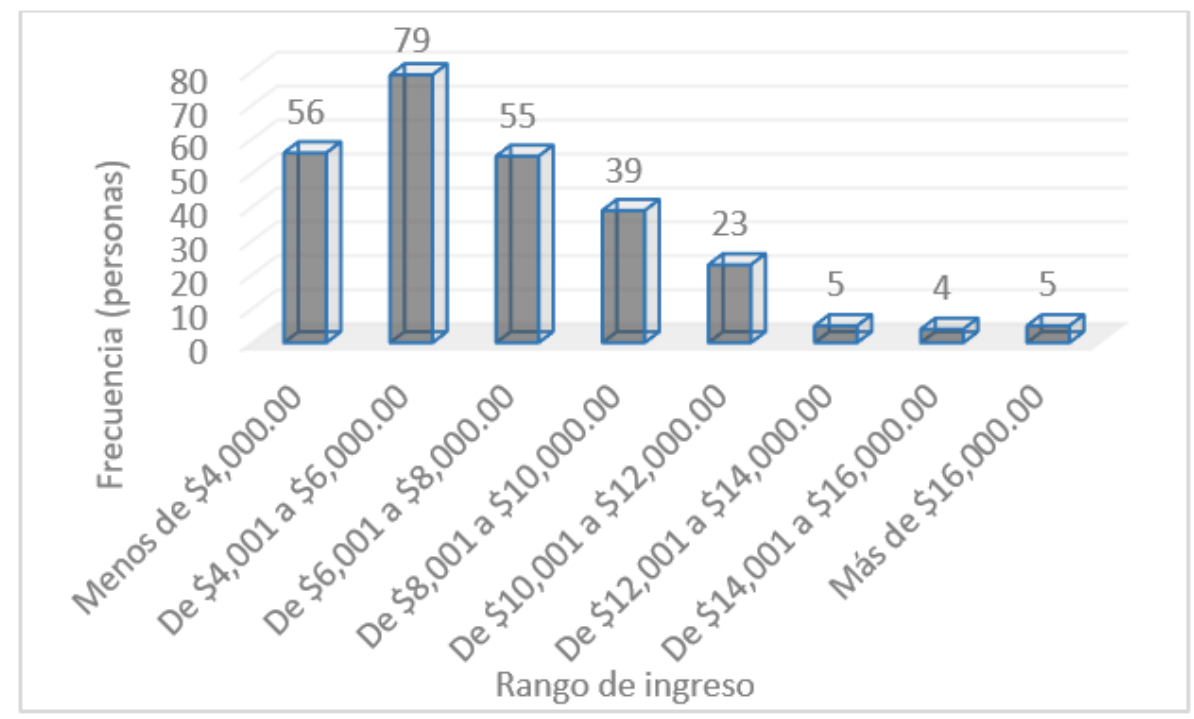

FIGURA 2.

Frecuencia del ingreso.

Elaboración con base a las encuestas.

El ingreso de la población encuestada en promedio fue de $\$ 6,700.00$ mensuales. Se agruparon en 8 rangos para efectos de analizar dónde se concentró el ingreso. El $71 \%$ de la población declaró un ingreso menor o igual de $\$ 8,000.00$ mensuales $(21.1 \%$ menos de $\$ 4,000.00$, el $29.70 \%$ entre $\$ 4001.00$ y $\$ 6,000.00$ y el $20.68 \%$ entre $\$ 6001.00$ y $\$ 8,000.00$ mensuales). Menos del $2 \%$ de la población dijo percibir un ingreso mensual mayor a $\$ 16,000.00$ (figura 2).

Con base al análisis descriptivo de las variables, el 65\% de la población encuestada mostró una disponibilidad al pago para conservar los bosques que se encuentran en la parte alta de la cuenca; un $28.9 \%$ no está dispuesta a pagar por motivos de desconfianza a una mala administración del recurso. El 88\% percibe que el medio ambiente donde vive está deteriorado, los recursos en los que consideran un alto deterioro son: agua con $63.7 \%$, aire con $57.1 \%$ y bosque con $45.1 \%$. Así mismo el $85 \%$ dijo conocer los beneficios que el bosque proporciona.

\section{Análisis econométrico}

Las variables más significativas se identificaron con base a la distribución logística del modelo propuesto. El modelo que se estimó se expresó como:

Interpretación de los coeficientes de las variables: las variables con coeficientes negativos indican una relación inversa con la variable dependiente (DAP) tal es el caso de la: MAP1, EDA, EDU, GEN y TF, que a medida que se dé un aumento en estas variables, la disponibilidad de pago disminuye. A excepción de la variable EDU donde se esperaba un signo positivo, las demás variables son consistentes con lo que se esperaba. Las variables que tienen una relación directa con la DAP son: ING, PA y BAM, mayor ingreso mayor probabilidad de pago; si la percepción del deterioro aumenta, aumenta la DAP; a mayor conocimiento de los beneficios del bosque, existe una disponibilidad de un pago mayor (Cuadro 3). 
CUADRO 3.

Parámetros obtenidos para el modelo econométrico.

\begin{tabular}{llllll}
\hline Variable & Coeficiente & $\begin{array}{l}\text { Desviación } \\
\text { estándar }\end{array}$ & b/St.Er. & $\mathrm{P}[|\mathrm{Z}|>\mathrm{z}] \mid$ & $\begin{array}{l}\text { Media de } \\
\mathrm{X}\end{array}$ \\
\hline Constante & 2.8727214 & 0.9540352 & 3.011 & 0.0026 & \\
MAP1 & -0.213012 & 0.1256512 & -1.695 & 0.09 & 1.2347206 \\
ING & 0.0480098 & 0.0963798 & 0.498 & 0.6184 & 2.8409091 \\
EDA & -0.3692 & 0.1376566 & -2.682 & 0.0073 & 3.0871212 \\
EDU* & -0.555304 & 0.1792547 & -3.098 & 0.0019 & 2.4356061 \\
GEN $^{*}$ & -0.679577 & 0.2922128 & -2.326 & 0.02 & 0.5075758 \\
TF & -0.393838 & 0.2282674 & -1.725 & 0.0845 & 1.7575758 \\
PA & 0.592703 & 0.4399741 & 1.347 & 0.1779 & 0.8825758 \\
BAM $^{*}$ & 1.1712002 & 0.3986107 & 2.938 & 0.0033 & 0.8560606 \\
\hline
\end{tabular}

laboración a partir de la salida del software N-Logit.

${ }^{*}$ Significancia al $5 \%$.

Las pruebas que se aplicaron para analizar la probabilidad de pago para conservar el bosque mediante el monto estimado fueron: Predicción del modelo. El modelo predice correctamente 189 (34+155) observaciones o $71.6 \%$, lo que significa que la predicción es admisible. Ajuste del modelo. Se utilizó la pseudo $\overline{\mathrm{R}}^{2}$ de McFadden con valor de 0.12 , un valor mayor 0.10 se considera aceptable (Bateman et al, 2002). Prueba

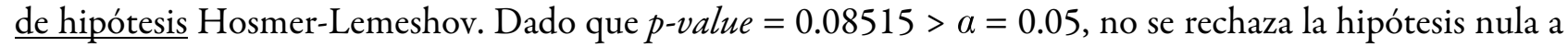
una confiabilidad del $95 \%$, por lo que se concluye que las predicciones del modelo no difieren de los valores observados, reflejando un buen ajuste. $\underline{Y}$ Test de la razón de verosimilitud (LR) es de 41.8752 y su $\rho$ value es de cero, por lo que una confiabilidad del $95 \%$ se rechaza la hipótesis nula a favor de la alterna, dado que $(p$-vlaue $<=0.05)$, por lo tanto, se concluye que por lo menos un parámetro tiene que ser diferente de cero (Cuadro 4).

CUADRO 4.

Estimación de parámetros por máxima verosimilitud.

\begin{tabular}{ll}
\hline Variable dependiente & DAP \\
\hline Variable de peso & Ninguna \\
Numero de observaciones & 264 \\
Iteraciones completadas & 5 \\
Función de máxima verosimilitud & -149.1053 \\
Numero de parámetros & 9 \\
Criterio de información: AIC $=$ & 1.19777 \\
Muestra finita AIC & 1.20045 \\
Criterio de información: BIC = & 1.31967 \\
Criterio de información: HQIC & 1.24675 \\
Máxima verosimilitud restringida & -170.0429 \\
Pseudo-R2 de McFadden & 0.1231312 \\
Chi cuadrada & 41.87518 \\
Grados de libertad & 8 \\
Prob[Chi cuadrada $>$ valor] $=$ & $1.43 \mathrm{E}-06$ \\
Chi cuadrada Hosmer-Lemeshow = & 13.8725 \\
p-value = 0.08515 con 8 grados de libertad
\end{tabular}

Elaboración a partir de la salida del software N-Logit. 


\section{Efectos marginales de las variables}

Los cambios marginales en la probabilidad asociados a la variable dependiente (DAP) con respecto a cada una de las variables explicativas, manteniéndose constantes el resto de las variables, se interpreta como el cambio producido en la DAP, ante un cambio de la variable. El conocimiento que los encuestados tienen de los beneficios que proporciona el bosque (BAM) es la variable más influyente en la probabilidad de un pago para conservar el bosque, la probabilidad cambia en $27.8 \%$ a medida que aumenta el conocimiento de los encuestados (Cuadro 5).

CUADRO 5

Efectos marginales

\begin{tabular}{ll}
\hline Variable & Efecto marginal \\
\hline Constante & 0.62765 \\
MAP1 & -0.04654 \\
ING & 0.01049 \\
EDA & -0.08066 \\
EDU & -0.12133 \\
GEN & -0.14733 \\
TF & -0.08605 \\
PA & 0.13793 \\
BAM & 0.27789 \\
\hline
\end{tabular}

Elaboración a partir de la salida del software N-Logit.

\section{Análisis económico}

La DAP promedio por persona es de $\$ 13.33$ mensual calculada a partir de 264 observaciones, el valor económico del bosque se obtuvo multiplicando la DAP promedio por la PEA de la región estudio $(40,418)$, lo que equivale a $\$ 539,111.45$ por mes, este monto es en lo que se estima la conservación del bosque de la parte alta de la cuenca de estudio (Cuadro 6).

CUADRO 6.

Estimación de la DAP.

\begin{tabular}{ll}
\hline Descripción & Monto \\
\hline DAP por mes/persona & $\$ 13.34$ \\
Valor por mes en la región de & $\$ 539,111.45$ \\
estudio & $\begin{array}{l}\text { Valor total anual en la región } \\
\text { de estudio }\end{array}$ \\
\hline
\end{tabular}

Elaboración a partir de la salida del software N-Logit.

\section{Conclusiones}

Existe una disponibilidad de pago positiva por conservar los bosques que se encuentra en la parte alta de la cuenca. El 65\% de los encuestados está dispuesto a pagar por conservar el bosque.

La DAP por persona por mes es de $\$ 13.33$. El valor económico del bosque se estimó en $\$ 539,111.45$ por mes, equivalente a $\$ 6,469,337.41$ anual. 
El 85\% de los encuestados conoce los beneficios que provee el bosque; el $88 \%$ percibe que el ambiente donde vive está deteriorado. Los recursos que tienen un deterioro alto son el: agua, aire y bosque.

Las variables que influyeron de manera significativas para determinar la DAP fueron: la edad, la educación, el género y el conocimiento que los encuestados tienen de los beneficios que genera el bosque. La población que encuentra en edad productiva mostró mayor interés por cuidar los bosques.

El ingreso, la percepción ambiental y el conocimiento de los beneficios que provee el bosque tienen relación positiva con la DAP.

El poder adquisitivo de la población encuestada es un factor que influye en la disposición a pagar, a mayor ingreso mayor disponibilidad a pagar.

\section{LITERATURA CITADA}

Bateman, I. J., Carson, R. T., Day, B., Hanemann, M., Hanley, N., Hett, T., Jones, L. M., Loomes, G., Mourato, S., Özdemirog, Lu. E., Pearce, D.W., Sugden, R. y Swason, J., (2002). Economic valuation with stated preference techniques: a manual. Edward Elgar Publishing. Cheltenham, UK, England. https:// doi.org/10.4337/9781781009727

Bishop, R. C. y Heberlein, T. A. (1979). Measuring values of extramarket goods: Are indirect measures biased? American journal of agricultural economics, 61, 926-930. https://doi.org/10.2307/3180348

Comisión Nacional del Agua (2009). Estadísticas del agua de la Región Hidrológico-Administrativa XIII, aguas del valle de México.

Del Ángel Pérez, A. L., Rebolledo Martínez. A., Villagómez Cortés. J. A. y Zetina Lezama, R. (2009). Valoración del servicio ambiental hidrológico en el sector doméstico de San Andrés Tuxtla, Veracruz, México. Estudios sociales (Hermosillo, Son.), 17(33), 225-257.

Haab, T. C. y McConnell, K. E. (2002). Valuing environmental and natural resources: the econometrics of non-market valuation. Edward Elgar Publishing. https://doi.org/10.4337/9781843765431

Hanemann, W. M. (1984). Welfare evaluations in contingent valuation experiments with discrete responses. American journal of agricultural economics, 66(3), 332-341. https://doi.org/10.2307/1242685

Landell-Mills, N. y Porras, I. T. (2002). Silver bullet or fools' gold?: a global review of markets for forest environmental services and their impact on the poor. London: International Institute for Environment and Development. London.

Martínez Parra, J. (2011). Actores claves en la consolidación de la base social: cuenca de la laguna de Tecocomulco. Comisión de Cuenca de la Laguna de Tecocomulco.

Riera, P. (1994). Manual de valoración contingente. Ministerio de Economía y Hacienda, Instituto de Estudios Fiscales. http://132.247.70.26/profesores/blopez/valoracion-manual.pdf.

Saldívar V., A., Olivera V., M. y Isidro C., A. (2013). Valoración y demanda del servicio ambiental hidrológico en el Parque Nacional Cumbres de Monterrey. Revista Natura@economía, 1(2), 9-28.

Secretaría de Medio Ambiente y Recursos Naturales (2016). Informe de la situación del medio ambiente en México. Compendio de Estadísticas Ambientales, Indicadores Clave, de Desempeño Ambiental y de Crecimiento Verde. https://apps1.semarnat.gob.mx:8443/dgeia/informe15/tema/pdf/Informe15_completo.pdf.

Valdivia Alcalá, R.., Abelino Torres, G., López-Santiago, M. A. y Zavala Pineda, M. J. (2012). Valoración económica del reciclaje de desechos urbanos. Revista Chapingo. Serie ciencias forestales y del ambiente, 18(3), 435-447. https://doi.org/10.5154/r.rchscfa.2010.07.044

Vásquez Lavín, F., Cerda Urrutia, A. y Orrego Suaza, A. (2007). Valoración económica del ambiente: Fundamentos Económicos, Econométricos y Aplicaciones. Buenos Aires: Thomson Learning. 